\title{
Zinc Catalyzed Claisen Rearrangement of Allyl Aryl Ethers to o-Allylated Phenols in Liquid Phase
}

\author{
Monika Gupta*
}

Department of Chemistry, University of Jammu, Jammu-180006, India

\begin{abstract}
A simple and efficient method is developed for the zinc catalyzed Claisen rearrangement of allyl aryl ethers to $o$-allyl phenols by stirring in an oil-bath at $55^{\circ} \mathrm{C}$ in liquid phase. Moreover, zinc powder is re-cyclable up to six times use without much loss of significant activity. In addition to it, the products are obtained in good to excellent yields and are in a state of high purity. The structures of the products are confirmed by ${ }^{1} \mathrm{H}$ NMR, ${ }^{13} \mathrm{CNMR}$, IR and mass spectral data and comparison with authentic samples prepared according to the literature methods.
\end{abstract}

Keywords: Allyl aryl ethers, $o$-allyl phenols, zinc powder, THF, recyclable.

\section{INTRODUCTION}

Claisen rearrangement [1-3] finds numerous applications in the biosynthesis of natural products [4], such as fatty acids and polyketides. It occupies a unique place in the biomimetic total synthesis of desoxymorellin [5], and forbesione [6] which proceeds in a regioselective manner and produces desired scaffold exclusively like hanburin [7], morellin [8] and bractatins [9].

Ethers are very routinely used as protecting groups for hydroxyl functions during organic synthesis and besides a large number of ethers are known as good blocking groups [10], the role of aliphatic, benzylic and allylic ethers are particularly noteworthy. The conversion of ethers to phenols can be performed in numerous ways, although the success of the transformation largely depends on the judicious choice [11] of the deblocking agent that is compatible with other functional groups present in the ether molecule. Numerous reagents are available for the rearrangement of ethers to corresponding phenols such as bismuth triflate $[12,13]$, chiral lewis acid $[14,15]$, orthometallated complex(R,R)-di$\mu$-chlorobis $\{9-[(1-$ dimethylamino $)$ ethyl $]-10$-phenanthrenyl-C,N $\}$ dipalladium [16], chiral aluminium reagent [17] and KHMDS [18]. But most of the reported reagents are associated with certain drawbacks such as expensiveness, toxic nature and tedious work-up procedure. Thus, there remains a need to find a new environmentally benign procedure, which should be simple, safe and economic for Claisen rearrangement of allyl aryl ethers.

Since zinc powder is non-toxic and inexpensive. It has been used for selective $\alpha$-haloacylation and gem -bisacylation of alkyl aldehydes [19], synthesis of $o$-arylene dizinc compounds [20], synthesis of benzhydrols [21], transesterification of $\beta$ ketoesters [22], acylation and sulfonation of pyrrole [23], Fries rearrangement [24] and in Williamson synthesis of ethers [25]. Moreover, zinc oxide, a versatile reagent for benzylic oxidations has also been reported [26].

*Address correspondence to this author at the Department of Chemistry, University of Jammu, Jammu-180006, India; Tel: 9419623841, 2437775; Email: monika.gupta77@indiatimes.com
Keeping in view the importance of Claisen rearrangement of allyl aryl ethers in organic synthesis and our continued interest in devising environmentally benign methodologies, we have successfully developed a zinc catalyzed synthesis of $o$-allylated phenols in liquid phase. This method is superior to already existing methods as there is no need of toxic and expensive reagents, work-up being simply reduced to filteration followed by treatment with crushed ice.

\section{RESULTS AND DISCUSSION}

The experimental conditions were optimized to proceed the reaction safely and in milder conditions. The amount of catalyst used was found to be crucial to proceed the reaction faster and in environment- friendly way. After carrying out the reaction under different set of conditions, the optimum conditions selected were: for 10 mmole of allyl aryl ether, 2.5 mmole of zinc powder was required. In a test reaction, to allyl phenyl ether $(10 \mathrm{mmol})$ and zinc powder $(2.5 \mathrm{mmol})$, THF $(5 \mathrm{~mL})$ was added and stirred at $55^{\circ} \mathrm{C}$, product was isolated in $80 \%$ yield in $40 \mathrm{~min}$. (Scheme 1).

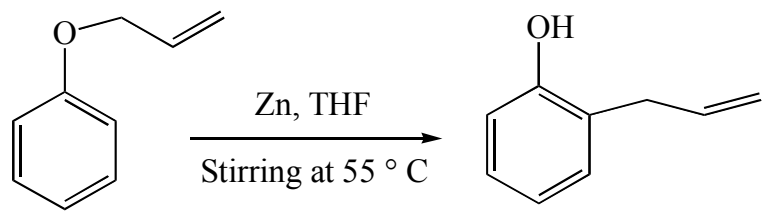

Scheme 1.

To the best of our knowledge, this type of Claisen rearrangement of allyl aryl ethers in the presence of zinc powder is unprecedented. This prompted us to investigate this unusual $\mathrm{Zn}$ catalyzed synthesis of $o$-allylated phenols. It was found that zinc has not yet been used for such rearrangement under milder conditions. This rearrangement was also compared with the reported reagent such as orthometallated complex $(\mathrm{R}, \mathrm{R})$-di- $\mu$-chlorobis $\{9$-[(1-dimethylamino)ethyl]-10-phenanthrenyl-C,N $\}$ dipalladium in case of allyl aryl ether and found that the reaction took more time, lesser yield, work-up procedure was tedious and moreover, the expensive nature of the reagent made the 
Table1. Results of Zinc Powder Catalyzed Claisen Rearrangement of Allyl Aryl Ethers to o-Allylated Phenols in THF

\begin{tabular}{|c|c|c|c|c|}
\hline Entry & Reactant & Product $^{\mathrm{a}}$ & Time (min.) & Yield $^{\mathrm{b}}(\%)$ \\
\hline 1. & Allyl phenyl ether & 2-Allyl phenol & 40 & 80 \\
\hline 3. & Allyl(4-methylphenyl) ether & 2-Allyl4-methylphenol & 75 & 93 \\
\hline 4. & Allyl(4-formylphenyl) ether & 2-Ally14-formylphenol & 60 & 85 \\
\hline 6. & Allyl(4-formyl-2-methoxyphenyl)ether & 6-Allyl2-methoxy-4-formyl phenol & 120 & 78 \\
\hline 7. & Allyl(4-nitrophenyl)ether & 2-Allyl4-nitrophenol & 25 & 83 \\
\hline 8. & Allyl(4-bromophenyl)ether & 2-Allyl4-bromophenol & 50 & 78 \\
\hline 9. & Allyl(4-methoxy phenyl) ether & 2-Allyl4-methoxy phenol & 70 & 70 \\
\hline
\end{tabular}

${ }^{\mathrm{a}}$ All the products are liquids and purified by passing through a column of silica gel and elution with ethylacetate and petroleum ether (1:2). These products were characterized by ${ }^{1} \mathrm{H}$ NMR, ${ }^{13} \mathrm{CNMR}$, IR and mass spectral data.

${ }^{\mathrm{b}}$ Yields refer to the isolated yields.

process highly expensive while the reaction with easily available zinc powder can be carried out under mild reaction conditions. Moreover, work-up procedure is simple and recyclability of the catalyst makes the process cost-effective.

To demonstrate the versatility of reagent, a series of allyl aryl ethers substituted with electron-releasing and electronwithdrawing groups were subjected to Claisen rearrangement under the similar reaction conditions, products were obtained in good to excellent yields as shown in Table $\mathbf{1}$.

The plausible mechanism involved is the ionic mechanism and it is expected that lone pair of electrons from oxygen atom gets interacted with zinc generating a polar transition state. Then pi electrons of allyl shifted giving positive charge on the end terminal carbon providing the driving force to balance the partial $+v e$ charge on oxygen atom. O-Zn bond cannot stay for longer time. It gets broken with the result $\mathrm{C}=\mathrm{O}$ is formed shifting the pi electrons of the ring towards positive centre of allyl group and finally the aromaticity of the ring is maintained by shifting the $\mathrm{H}$ atom thereby converting $\mathrm{C}=\mathrm{O}$ to $\mathrm{C}-\mathrm{OH}$.

Another important feature of zinc powder lies in the fact that after carrying out the reaction using optimum conditions, product was isolated after the separation of zinc powder. This much amount of zinc powder, which is left after first use, can be re-used up to six times after simple washing with diethyl ether and dilute HCL without much loss of activity, thus rendering the process still more economic (Table 2). Catalytic activity goes on decreasing after every use which can be shown in Table 2 but the zinc cannot be regenerated for re-use.

\section{CONCLUSIONS}

In conclusion, we have developed a $\mathrm{Zn}$ catalyzed procedure for very simple and efficient Claisen rearrangement of allyl aryl ethers to corresponding $O$ allylated phenols by stirring in an oil-bath at $55^{\circ} \mathrm{C}$ and the products are isolated in excellent yields and are in a state of high purity. Moreover, zinc powder can be re-cycled up to six times without much loss of significant activity that makes the process cost-effective.
Table 2. Results of Re-Use Studies of Claisen Rearrangement in the Case of Entry 1 Using Zinc Powder by Stirring at $55^{\circ} \mathrm{C}$

\begin{tabular}{|c|c|c|c|}
\hline Entry $^{\mathbf{a}}$ & No. of Uses & Time (min.) & Yield $\left.^{\mathbf{b}} \mathbf{\%}\right)$ \\
\hline \hline 1. & 1 & 40 & 80 \\
\hline $\mathbf{1 .}$ & 2 & 55 & 79 \\
\hline $\mathbf{1 .}$ & 3 & 72 & 76 \\
\hline $\mathbf{1 .}$ & 4 & 80 & 72 \\
\hline $\mathbf{1 .}$ & 5 & 85 & 70 \\
\hline $\mathbf{1 .}$ & 6 & 92 & 66 \\
\hline
\end{tabular}

${ }^{\mathrm{a} I n}$ case of entry 1, reaction optimum conditions are: for allyl phenyl ether $(10 \mathrm{mmol})$ and zinc powder $(2.5 \mathrm{mmol})$, THF $(5 \mathrm{~mL})$ was required. The reaction mixture was stirred at $55^{\circ} \mathrm{C}$. The reaction was completed in $40 \mathrm{~min}$., then product was isolated in $80 \%$ yield by distilled off the solvent.

${ }^{\mathrm{b}} \mathrm{Yields}$ refer to the isolated yields.

\section{EXPERIMENTAL SECTION}

\section{General}

All the melting points were determined on a Perfit melting point apparatus and are uncorrected. ${ }^{1} \mathrm{H}$ NMR and ${ }^{13}$ CNMR spectra were recorded on a Bruker DPX-200 NMR spectrometer $(200 \mathrm{MHz})$ in $\mathrm{CDCl}_{3}$ using tetramethyl silane as internal standard and IR spectra was recorded using $\mathrm{KBr}$ disc on a Perkin Elmer FTIR spectrometer. The mass spectral data was obtained on Bruker Esquires 3000 (ESI). The reactions were monitored qualitatively by TLC.

\section{General Procedure for the Zinc Powder Catalyzed Claisen Rearrangement of Allyl Aryl Ethers to o-Allyl Phenols}

Allyl aryl ether $(10 \mathrm{mmol})$, zinc powder $(2.5 \mathrm{mmol})$ and THF $(5 \mathrm{~mL})$ were taken in a round bottomed flask $(25 \mathrm{~mL})$ The reaction mixture was stirred in an oil-bath at $55^{\circ} \mathrm{C}$ for the required time. After completion of the reaction (monitored by TLC, visualization in iodine vapours), filtered off the reaction mixture so as to remove the zinc powder. 
Now, the solvent was distilled off and the product was isolated in good to excellent yields. The products were purified by crystallization through a column of silica gel and elution with ethyl acetate and petroleum ether $(1: 2)$.

The structures of the products were confirmed by IR, ${ }^{1} \mathrm{H}$ NMR, ${ }^{13}$ CNMR and mass spectral data.

\section{Spectral Data of the Synthesized Compounds (1-10)}

Entry 1. IR ( $\mathrm{v}_{\max }$ in $\mathrm{cm}^{-1}, \mathrm{KBr}$ ): 3500, 3020, 2925, 1000; ${ }^{1} \mathrm{H}$ NMR $\left(\mathrm{CDCl}_{3}\right): \delta 3.22\left(\mathrm{~d}, 2 \mathrm{H}, \mathrm{CH}_{2}\right), 4.93-4.96(\mathrm{~m}, 2 \mathrm{H}$, $\left.=\mathrm{CH}_{2}\right), 5.00(\mathrm{~s}, 1 \mathrm{H}, \mathrm{OH}), 6.30(\mathrm{~d}, 1 \mathrm{H},=\mathrm{CH}), 6.60-6.70(\mathrm{~m}$, 2H, Harom), 6.80-6.90 (m, 2H, Harom); ${ }^{13}$ CNMR $\left(\mathbf{C D C l}_{3}\right)$ : $\delta$ 137.5(-CH), 136.4(C-1), $134.2(\mathrm{C}-4), 129.5(\mathrm{C}-2), 128.5$ $(\mathrm{C}-5,6), \quad 128.4\left(=\mathrm{CH}_{2}\right), \quad 128(\mathrm{C}-3), 29.1\left(-\mathrm{CH}_{2}\right) ;$ Mass: $\mathrm{m} / \mathrm{z}$ $\left(\mathrm{M}^{+}\right): 134$

Entry 2. IR ( $\mathrm{v}_{\max }$ in $\left.\mathrm{cm}^{-1}, \mathrm{KBr}\right): 3500,3020,2925,1000$, $728 ;{ }^{1} \mathrm{H}$ NMR $\left(\mathrm{CDCl}_{3}\right): \delta 3.22\left(\mathrm{~d}, 2 \mathrm{H}, \mathrm{CH}_{2}\right), 4.93-4.96(\mathrm{~m}$, $\left.2 \mathrm{H},=\mathrm{CH}_{2}\right), 5.00(\mathrm{~s}, 1 \mathrm{H}, \mathrm{OH}), 6.30(\mathrm{~d}, 1 \mathrm{H},=\mathrm{CH}), 6.55-6.60$ (m, 1H, Harom), 6.85-6.95 (m, 2H, Harom); ${ }^{13}$ CNMR $\left(\mathbf{C D C l}_{3}\right): \delta 150$ (C-4), 137.5(-CH), 136.4(C-1), 129.5(C-2), 128.5 (C-5,6), 128.4 $\left(=\mathrm{CH}_{2}\right), 128(\mathrm{C}-3), 29.1\left(-\mathrm{CH}_{2}\right)$; Mass: $\mathrm{m} / \mathrm{z}\left(\mathrm{M}^{+}\right): 168.5$

Entry 3. IR ( $v_{\max }$ in $\left.\mathrm{cm}^{-1}, \mathrm{KBr}\right): 3500,3020,2925,1000$; ${ }^{1} \mathrm{H} \operatorname{NMR}\left(\mathrm{CDCl}_{3}\right): \delta 2.35\left(\mathrm{~s}, 3 \mathrm{H}, \mathrm{CH}_{3}\right), 3.22\left(\mathrm{~d}, 2 \mathrm{H}, \mathrm{CH}_{2}\right)$, 4.93-4.96 (m, $\left.2 \mathrm{H},=\mathrm{CH}_{2}\right), 5.00(\mathrm{~s}, 1 \mathrm{H}, \mathrm{OH}), 6.30(\mathrm{~d}, 1 \mathrm{H}$, $=\mathrm{CH}), \quad 6.45-6.52(\mathrm{~m}, 1 \mathrm{H}$, Harom $), 6.65-6.70(\mathrm{~m}, 2 \mathrm{H}$, Harom); ${ }^{13}$ CNMR (CDCl $)_{3}$ : $\delta$ 137.5(-CH), 136.4(C-1), 129.5(C-2), $128.5(\mathrm{C}-5,6), 128.4\left(=\mathrm{CH}_{2}\right), 128(\mathrm{C}-3), 127(\mathrm{C}-$ 4), 29.1(- $\left.\mathrm{CH}_{2}\right)$; Mass: $\mathrm{m} / \mathrm{z}\left(\mathrm{M}^{+}\right): 148$

Entry 4. IR ( $v_{\max }$ in $\left.\mathrm{cm}^{-1}, \mathrm{KBr}\right): 3600,3020,2925,1690$, $1000 ;{ }^{1} \mathrm{H}$ NMR $\left(\mathrm{CDCl}_{3}\right): \delta 3.22\left(\mathrm{~d}, 2 \mathrm{H}, \mathrm{CH}_{2}\right), 4.93-4.96(\mathrm{~m}$, $\left.2 \mathrm{H},=\mathrm{CH}_{2}\right), 5.00(\mathrm{~s}, 1 \mathrm{H}, \mathrm{OH}), 6.30(\mathrm{~d}, 1 \mathrm{H},=\mathrm{CH}), 6.75-6.80$ (m, 1H, Harom), 7.38-7.45 (m, 2H, Harom), $9.87(\mathrm{~s}, 1 \mathrm{H}$, CHO) ${ }^{3}{ }^{3}$ CNMR $\left(\mathbf{C D C l}_{3}\right): \delta$ 137.5(-CH), 136.4(C-1), 132(C4), 129.5(C-2), $128.5(\mathrm{C}-5,6), 128.4\left(=\mathrm{CH}_{2}\right), 128(\mathrm{C}-3), 29.1(-$ $\left.\mathrm{CH}_{2}\right) ;$ Mass: $\mathrm{m} / \mathrm{z}\left(\mathrm{M}^{+}\right): 163$.

Entry 5. IR ( $v_{\max }$ in $\left.\mathrm{cm}^{-1}, \mathrm{KBr}\right): 3600,3020,2925,1715$, $1000 ;{ }^{1} \mathrm{H}$ NMR $\left(\mathrm{CDCl}_{3}\right): \delta 2.55\left(\mathrm{~s}, 3 \mathrm{H}, \mathrm{COCH}_{3}\right), 3.22(\mathrm{~d}$, $\left.2 \mathrm{H}, \mathrm{CH}_{2}\right), 4.93-4.96\left(\mathrm{~m}, 2 \mathrm{H},=\mathrm{CH}_{2}\right), 5.00(\mathrm{~s}, 1 \mathrm{H}, \mathrm{OH}), 6.30$ $(\mathrm{d}, 1 \mathrm{H},=\mathrm{CH}), 6.70-6.75(\mathrm{~m}, 1 \mathrm{H}$, Harom $), 7.45-7.55(\mathrm{~m}, 2 \mathrm{H}$, Harom); ${ }^{13}$ CNMR $\left(\mathbf{C D C l}_{3}\right): \delta \quad 197.6(\mathrm{CO})$ 137.5(-CH), 136.4(C-1), 132(C-4), 129.5(C-2), $128.5 \quad$ (C-5,6), 128.4 $\left(=\mathrm{CH}_{2}\right), 128(\mathrm{C}-3), 29.1\left(-\mathrm{CH}_{2}\right), 26.3\left(-\mathrm{CH}_{3}\right) ;$ Mass: $\mathrm{m} / \mathrm{z}$ $\left(\mathrm{M}^{+}\right): 176$.

Entry 6. IR ( $v_{\max }$ in $\left.\mathrm{cm}^{-1}, \mathrm{KBr}\right): 3600,3020,2925,1690$, 1060, 1000; ${ }^{1} \mathrm{H}$ NMR $\left(\mathrm{CDCl}_{3}\right): 3.22\left(\mathrm{~d}, 2 \mathrm{H}, \mathrm{CH}_{2}\right), 3.92$ (s, $\left.3 \mathrm{H}, \mathrm{OCH}_{3}\right), 4.93-4.96\left(\mathrm{~m}, 2 \mathrm{H},=\mathrm{CH}_{2}\right), 5.00(\mathrm{~s}, 1 \mathrm{H}, \mathrm{OH}), 6.30$ $(\mathrm{d}, 1 \mathrm{H},=\mathrm{CH}), 6.75-6.80,6.95-7.00(\mathrm{~m}, 1 \mathrm{H}$, Harom $), 7.38-$ 7.45, $9.87(\mathrm{~s}, 1 \mathrm{H}, \mathrm{CHO}) ;(\mathrm{m}, 2 \mathrm{H}$, Harom $), 9.87(\mathrm{~s}, 1 \mathrm{H}$, CHO); ${ }^{13}$ CNMR:(CDCl 3$): \delta \quad 137.5(-\mathrm{CH}), \quad 136.4(\mathrm{C}-1)$, 132(C-4), 129.5(C-2), $128.5(\mathrm{C}-5,6), 128.4\left(=\mathrm{CH}_{2}\right), 128(\mathrm{C}-$ 3), $55\left(\mathrm{OCH}_{3}\right), 29.1\left(-\mathrm{CH}_{2}\right)$; Mass: $\mathrm{m} / \mathrm{z}\left(\mathrm{M}^{+}\right): 191$.

Entry 7. IR ( $v_{\max }$ in $\left.\mathrm{cm}^{-1}, \mathrm{KBr}\right): 3500,3020,2925,1440$, 1000; ${ }^{1} \mathrm{H} \mathrm{NMR}\left(\mathrm{CDCl}_{3}\right): \delta 3.22\left(\mathrm{~d}, 2 \mathrm{H}, \mathrm{CH}_{2}\right), 4.93-4.96(\mathrm{~m}$, $\left.2 \mathrm{H},=\mathrm{CH}_{2}\right), 5.00(\mathrm{~s}, 1 \mathrm{H}, \mathrm{OH}), 6.30(\mathrm{~d}, 1 \mathrm{H},=\mathrm{CH}), 6.45-6.52$ (m, 1H, Harom), 6.65-6.70 (m, 2H, Harom); ${ }^{13} \mathrm{CNMR}$ $\left(\mathbf{C D C l}_{3}\right): \delta 138.3(\mathrm{C}-4), 137.5(-\mathrm{CH}), 136.4(\mathrm{C}-1), 129.5(\mathrm{C}-2)$, $128.5(\mathrm{C}-5,6), 128.4\left(=\mathrm{CH}_{2}\right), 128(\mathrm{C}-3), 29.1\left(-\mathrm{CH}_{2}\right)$; Mass: $\mathrm{m} / \mathrm{z}\left(\mathrm{M}^{+}\right): 180$
Entry 8. IR ( $\mathrm{v}_{\max }$ in $\left.\mathrm{cm}^{-1}, \mathrm{KBr}\right): 3500,3020,2925,1000$, 838; ${ }^{1} \mathrm{H}$ NMR $\left(\mathrm{CDCl}_{3}\right): \delta 2.35\left(\mathrm{~s}, 3 \mathrm{H}, \mathrm{CH}_{3}\right), 3.22(\mathrm{~d}, 2 \mathrm{H}$, $\left.\mathrm{CH}_{2}\right), 4.93-4.96\left(\mathrm{~m}, 2 \mathrm{H},=\mathrm{CH}_{2}\right), 5.00(\mathrm{~s}, 1 \mathrm{H}, \mathrm{OH}), 6.30(\mathrm{~d}$, $1 \mathrm{H},=\mathrm{CH}), 6.45-6.52(\mathrm{~m}, 1 \mathrm{H}$, Harom $), 6.65-6.70(\mathrm{~m}, 2 \mathrm{H}$, Harom); ${ }^{13}$ CNMR (CDCl $\left.)_{3}\right) \delta 140 \quad(\mathrm{C}-4), 137.5(-\mathrm{CH})$, 136.4(C-1), 129.5(C-2), $128.5(\mathrm{C}-5,6), 128.4\left(=\mathrm{CH}_{2}\right), 128(\mathrm{C}-$ 3), 29.1(- $\left.\mathrm{CH}_{2}\right)$; Mass: $\mathrm{m} / \mathrm{z}\left(\mathrm{M}^{+}\right): 212$

Entry 9. IR ( $v_{\max }$ in $\left.\mathrm{cm}^{-1}, \mathrm{KBr}\right): 3500,3020,2925,1275$, 1000; ${ }^{1} \mathrm{H} \operatorname{NMR}\left(\mathrm{CDCl}_{3}\right): \delta 3.22\left(\mathrm{~d}, 2 \mathrm{H}, \mathrm{CH}_{2}\right), 4.12(\mathrm{~s}, 3 \mathrm{H}$, $\left.\mathrm{CH}_{3}\right), 4.93-4.96\left(\mathrm{~m}, 2 \mathrm{H},=\mathrm{CH}_{2}\right), 5.00(\mathrm{~s}, 1 \mathrm{H}, \mathrm{OH}), 6.30(\mathrm{~d}$, $1 \mathrm{H},=\mathrm{CH}), 6.45-6.52(\mathrm{~m}, 1 \mathrm{H}$, Harom $), 6.65-6.70(\mathrm{~m}, 2 \mathrm{H}$, Harom); ); ${ }^{13}$ CNMR (CDCl $\left.)_{3}\right): \delta 160$ (C-4), 137.5(-CH), 136.4(C-1), 129.5(C-2), $128.5(\mathrm{C}-5,6), 128.4\left(=\mathrm{CH}_{2}\right), 128(\mathrm{C}-$ 3), 29.1 $\left(-\mathrm{CH}_{2}\right)$; Mass: $\mathrm{m} / \mathrm{z}\left(\mathrm{M}^{+}\right): 164$

Entry 10. IR ( $\mathrm{v}_{\max }$ in $\left.\mathrm{cm}^{-1}, \mathrm{KBr}\right): 3500,3020$, 2925,2800,1275, 1000; ${ }^{1} \mathrm{H} \operatorname{NMR}\left(\mathrm{CDCl}_{3}\right): \delta 2.28(\mathrm{t}, 3 \mathrm{H}$, $\left.\mathrm{CH}_{3}\right), 2.35\left(\mathrm{~s}, 3 \mathrm{H}, \mathrm{CH}_{3}\right), 3.22\left(\mathrm{~d}, 2 \mathrm{H}, \mathrm{CH}_{2}\right), 4.12(\mathrm{q}, 2 \mathrm{H}$, $\left.\mathrm{CH}_{2}\right), 4.93-4.96\left(\mathrm{~m}, 2 \mathrm{H},=\mathrm{CH}_{2}\right), 5.00(\mathrm{~s}, 1 \mathrm{H}, \mathrm{OH}), 6.30(\mathrm{~d}$, $1 \mathrm{H},=\mathrm{CH}), 6.45-6.52(\mathrm{~m}, 1 \mathrm{H}$, Harom $), 6.65-6.70(\mathrm{~m}, 2 \mathrm{H}$, Harom); ); ${ }^{13}$ CNMR(CDCl 3 ): $\delta 160$ (C-4), 137.5(-CH), 136.4(C-1), 129.5(C-2), $128.5(\mathrm{C}-5,6), 128.4\left(=\mathrm{CH}_{2}\right), 128(\mathrm{C}-$ 3), $60\left(\mathrm{OCH}_{2}\right), 29.1\left(-\mathrm{CH}_{2}\right), 13.8\left(\mathrm{OCH}_{2} \mathrm{CH}_{3}\right) ;$ Mass: $\mathrm{m} / \mathrm{z}$ $\left(\mathrm{M}^{+}\right): 178$

\section{ACKNOWLEDGEMENTS}

The author Monika Gupta (MG) is thankful to (CSIRUGC) for providing Senior Research Fellowship (SRF) during the period of research.

\section{REFERENCES}

[1] Naidu, R. Overview of Claisen rearrangement. Acc. Rapid Commun. Synth. Org. Chem., 2008, 4, 547.

[2] Sundararajan, G.; Ramesh, N. G. Unsaturated sugars: syntheses and applications. Molecules, 2005, 10, 833 .

[3] Lambert, T. H. Thesis submitted, California Institute of Technology, 2004

[4] Health, R. J.; Rock, C. O. Biomimetic self-condensation of malonates mediated by nucleosides. Nat. Prod. Rep., 2002, 19, 581

[5] Bhat, H. B.; Nair, P. M.; Venkataraman, K. Isoprenylated flavonoids - a survey. Indian J. Chem., 1974, 405.

[6] Leong, Y.-W.; Harrison, L. J.; Bennet, G. J.; Tan, H. T.-W. Regioselective synthesis of the bridged tricyclic core of garcinia Nat. Prod. J. Chem. Res. (S)., 1996, 372.

[7] Tisdale, E. J.; Slobodov, I.; Theodarkis, E.A. Cytotoxic caged tetraprenylated xanthonoids from Garcinia gaudichaudii (Guttiferae). Org. Biomol. Chem., 2003, 1, 4418.

[8] Asano, J.; Cliba, K.; Tada, M.; Yoshi, T. Claisen rearrangement in Xanthanoids. Phytochemistry, 1996, 41, 815.

[9] Rao, B. S. Total synthesis of seco-lateriflorone. J. Chem. Soc., 1937, 853 .

[10] (a) Flowers, H. M. Chemistry of the hydroxyl group ; Patai, S. Ed.; Interscience New York, 1969; Chapter 7, 1001 and references therein. (b) Cunnigham, J.; Warren, G. D. Study of metal and acid catalysed deprotection of propargyl ethers of alcohols via their allenyl ethers. Tetrahedron Lett., 1964, 1196.

[11] (a) Fujji, K.; Ichikawa, K.; Node, M.; Fuji, E. Enantioselective synthesis through enzymatic asymmetrization J. Org. Chem., 1970 44, 16. (b) Harrison, I. T.; Harrison, S. Compendium of Organic Method; Wiley Interscience: New York, 1971, 1, 92, 127. (c) Hanessian, S.; Sindon, Y. Inhibitors of interleukin-1.beta. converting enzyme. Tetrahedron Lett., 1980, 2305. (d) Jung, M. E.; Lyster, M. A. J. Org. Chem., 1977, 42, 3761. (e) Spyroudis, S.; Varvoglis, S. Claisen rearrangements. J. Chem. Soc. Chem. Comm., 1979, 615. (f) Narayan, C. R.; Iynger, K. N. Oxidation and reductions. J. Org. Chem., 1965, 30, 1734.

[12] Ollevier, T.; Mwene-Mbeja, T. M. Efficient and practical catalytic vinylogous aldol reaction of dioxinone-derived silyl dienol ethers with aromatic aldehydes. Tetrahedron Lett., 2006, 47, 4051. 
[13] Ollevier, T.; Deseroy, V.; Debailleul, B.; Vaur, S. Bismuth triflate, a selective reagent for Oxy-Cope Claisen rearrangement. Eur. J. Org. Chem., 2005, 4971.

[14] Ito, H.; Taguchi, T. Total synthesis of atroviridin. Chem. Soc. Rev., 1999, $28,43$.

[15] Majumdar, K. C.; Pal, A. K. Lewis acid catalyzed claisen rearrangement: regioselective synthesis of oxygen. Can. J. Chem., 2008, 73.

[16] Leung, P.-H.; Ng, K.-H.; Li, Y.; White, A. J. P.; Williams, D. J. catalytic asymmetric rearrangement of allylic trichloroacetimidates. a practical method for preparing allylic amines and congeners of high enantiomeric purity using orthometallated complex $(\mathrm{R}, \mathrm{R})$-di- $\mu-$ chlorobis $\{9-[(1-$ dimethylamino)ethyl]-10-phenanthrenyl-C,N $\}$ dipalladium. Chem. Commun., 1999, 2435.

[17] Mauoka, K.; Yamamoto, H. Enantioselective Claisen rearrangement. Synlett, 1991, 793.

[18] Kallmerten, J.; Gould, T. J. methods of Claisen rearrangement using KHMDS. J. Org. Chem., 1986, 51, 1152.

[19] Ishino, Y.; Mihara, M.; Takeuchi, T.; Takemoto, M. Zinc-metal promoted selective $\alpha$-haloacylation and gem-bisacylation of alkyl aldehydes in the presence of chlorotrimethylsilane. Tetrahedron Lett., 2004, 45, 3503.

[20] Amano, M.; Saiga, A.; Ikegami, R.; Ogata, T.; Takagi, K. Consecutive cross-coupling of o-phenylenedizinc compound with acyl and/or aryl halides in the presence of $\operatorname{Pd}(0)$-tris(2,4,6trimethoxyphenyl)phosphine. Tetrahedron Lett., 1998, 39, 8667.

[21] Ogawa, Y.; Saiga, A.; Mori, M.; Shibata, T.; Takegi, K Zincmediated novel and efficient method fo synthesis of benzhydrols. $J$. Org. Chem., 2000, 65, 1031.

[22] Chavan, S. P.; Shivasankar, K.; Sivappa, R.; Kale, R. Transesterification of $\beta$-ketoesters. Tetrahedron Lett., 2002, 43, 8583.

[23] Yadav, J. S.; Reddy, B. V.; Kondaji, G.; Rao, R. S.; Kumar, S. P. Acylation and sulfonation of pyrrole. Tetrahedron Lett., 2002, 43, 8133.

[24] Gupta, M.; Paul, S. Selective Fries rearrangement catalyzed by zinc powder. Synthesis, 2004, 11, 1789.

[25] Gupta, M.; Paul, S. Zinc catalyzed Williamson ether synthesis in the absence of base. Tetrahedron Lett., 2004, 45, 8825.

[26] Gupta, M.; Paul, S.; Gupta, R.; Loupy, A. Zinc oxide: A versatile reagent for benzylic oxidations. Tetrahedron Lett., 2005, 46, 4957.

(C) Monika Gupta; Licensee Bentham Open.

This is an open access article licensed under the terms of the Creative Commons Attribution Non-Commercial License (http://creativecommons.org/licenses/by-nc/ $3.0 /$ ) which permits unrestricted, non-commercial use, distribution and reproduction in any medium, provided the work is properly cited. 